\title{
Operative representation and the digital
}

\author{
G. Perin \\ School of Architecture, Faculty of Design, Architecture and Building, \\ University of Technology, Sydney
}

\begin{abstract}
Animation software promotes new architectural design practices where form making is presented as a 'clean' indexical translation of contextual data or information. The formal outcomes typical of this design method implicitly resist the foregrounding of representation, either as a depiction or a tool, as a subject worthy of research. Given the ubiquity of the image this rejection has a capacity to create a rupture between the profession and the wider community. The objective of this paper is to provide a critique of the implicit theoretical position animation software has to representation and explore how the application of other software can be used in conjunction with strategies and techniques aimed at a critical engagement with the usage of the image present within this broader social context. In doing so the discussion will draw on texts, including those of Robin Evans and W. J. T. Mitchell, as well as built projects and outcomes of design-based research.

The allure for abstract indexed form is evident of a longstanding architectural 'iconophobia' towards the image and as such embodies a very particular ideological bias that restricts the agency of architecture to contest its conventionalisation and subsequent deployment as a political tool or device of the market. In light of this an argument will be made for the generative capacity of the digital to act as a way of operatively engaging with the image's representational 'job'. The paper is not therefore opposed to the digital but against certain design practices that treat both the image as irrelevant and the modes of representation as an unproblematic and neutral transformative space.

Keywords: design methods, processes and creativity, digital design, representation and visualization, knowledge based design and generative systems, social aspects.
\end{abstract}




\section{Operative representation and the digital}

The adoption of animation software in architectural design practice has instigated a mode of form making that is promoted as a 'clean' indexical translation of contextual data. The argumentation accompanying such design action significantly recalibrates architecture's relationship to representation in two noteworthy ways. The first, somewhat paradoxically, normalises the instrumentality of the digital by imbuing animation software with such sophistication that the virtual space of the computer 'drawing' is rendered as a benign 'site' of transformation. The second is that the issue of figurative representation is explicitly condemned as an irrelevant and anachronistic architectural concern. Yet the abstract, non-representational projects emerging from this design practice seem inconsistent with the wider community's understanding of architecture, where the currency and ubiquity of the image unquestionably reinforces an expectation for it, via a figurative facility, to impart identity and meaning to the artefact. The paper, drawing primarily on my own post graduate design based research, aims to consider these conflicting ambitions for architecture as motivation to re-assess the role of representation in order to promote operative design strategies and techniques that engage critically with contemporary issues present within the public arena. Integral to this argument will be the promotion of a mode of digital design practice that openly acknowledges and exploits the image's figurative 'job' through the generative potential within all digital tools.

Undoubtedly the re-conception of representation from illustration and signification to generative tool offers a rare moment to re-think the processes and methods by which architecture is made. The instrumentality of these 'drawing' techniques regularise an experimental engagement released from the preoccupation to legitimise design action. Yet the argumentation for nonrepresentational form obviously witnesses a discomfort with representation and image as demonstrated in the following quote by Greg Lynn.

"The shift [...from meaning to machine...] is the primary explanation for the apparent alliance between certain aspects of Deleuze and Foucault's discourse and many contemporary architects now weary of representational critiques spanning from stylistic postmodernism to deconstruction..." Lynn [1].

This trivialising of representation can be interpreted as a criticism of the projection onto architectonic form the linguistic model underpinning Postmodern and Deconstruction theory. In making this point Lynn implicitly accepts that representation falls into such a framework, in as much as such projection can only be manifest as an imagistic symbolic device. Thus conceived of as an act of figurative labelling, the image's generative potential is trapped to the 'job' of attaining semiotic closure through a 'naming' or re-presenting.

It is possible to contextualise this semiotic limiting of the image as participating in a recurrent iconophobic architectural tradition where the desire for intellectual depth, often linked to a transcendental search, which asks the architect to go beyond the surface of things. In this light the shift from 'meaning' to 'machine' goes further than the machine's facility to abstract, 
process and reconfigure complex real world data. Drawing on W. J. T. Mitchell, this conventionalised interpretation of the image as a literal or 'natural' sign imparts an immediacy and directness that automatically evacuates the possibility that the image might occupy a generative space. In contrast Lynn's view of the 'machine' is more akin to Mitchell's discourse on the conventional status of the word, which acts as "...the artificial, arbitrary production of human will that disrupts natural presence by introducing unnatural elements into the world..." Mitchell [2]. Unlike the image which is wedded to that which it re-presents, animation software is deemed to be sophisticated because of its ability to productively respond and accommodate these artificial cultural constructs, and to do so through the use of process where form making is an emergent action rather than a pre-determined re-presentation.

As a consequence any potential for architecture to impart meaning resides only as a second order outcome. To quote Lynn again:

"The use of parameters and statistics for the design of form requires a more abstract, and often less representational origin for design. The shape of statistics, or parameters, may yield a culturally symbolic form, yet at the beginning, their role is more inchoate." Lynn [3].

Obviously the central architectural concern is the parametric capacity of animation software to capture the contingent and temporal. While careful to state that the digital is an artificial rather than organic process the trajectory of the argument constantly attempts to 'authenticate' this working method by associating the process with terms like 'natural' and 'evolutionary'. As Lynn writes "Animation... implies the evolution of a form and its shaping of forces; it suggests animalism, animism, growth, actualisation, vitality and virtuality..." Lynn [4].

This design practice is predicated on a geometric engagement with the contingencies of the real where static platonic form is replaced with the dynamic facility of the spline. Obviously, what is under scrutiny is the form and method of application rather than the issue of geometry itself. The promotion of the spline acts only as another manifestation of the desire to establish legitimacy through asserting a geometric underpinning the real. Therefore while one might tolerate such a correspondence on structural grounds the deployment of the spline to embody other forms of information serves only to complicate the status of the spline. Furthermore, the translation both through the mode of 'drawing', in the form of the diagram, and its material conversion questions the validity of such claims. In light of these realities the spline performs a representational 'job', by merely 'standing in' for information [5]. In keeping with Robin Evans's thesis, the mediating affect of the digital tool clearly locates its 'drawings' as a significant generative 'site' in the production of the artefact [6] (Translations from Drawing to Building best develop this thesis on the 'drawing'.)

The mediated nature of this information cannot, therefore purchase validity for the artefact. The unproblematic acceptance of information as valid or 'natural' also demonstrates a certain lack of criticality, preventing any contestation of either the status of the data and the worth of the temporal shifts it 
attempts to embody. Unable to fold in the complexity and deviation within the data this information is often converted into the statistical average, resulting in a privileging of the normative over the particular. The agency of the diagram in this process functions more akin to a kind of modernist reductive abstraction best identified by John Rajchman's in the essay Abstraction [7]. This reductive 'averaging' converts architecture into a prosthetic device, instituting an autonomous design process entrenched within the linear trajectory of a procedural logic. The jettisoning of the qualitative can therefore be seen to run counter to any claim of embedding the contingent and the temporal.

The erasure the impact in the process of translation simply re-sublimates the generative capacity of the tools and privilege instrumentality and production over any critical consideration affects or effects of or on the artefact. The contingency of the digital 'drawing' questions any investment made in text to establish such authenticity and instead necessitates a reconsideration of the potential of other software to index and process temporal information. This is not to deny that animation software still retains an architectural potency worthy of pursuit. The point is that the suppression of representation works only to restrict architecture's engagement with issues of signification and the generative capacity of all digital tools. Exactly at the moment when these tools offer a new space of experimentation, capable of addressing a wide range of architectural concerns, the compulsion for legitimacy threatens to conventionalise their deployment. (The sources of this anxiety are in the end unimportant in that they mask the effect of this compulsion.) This also converts the 'drawing' from a space of opportunist engagement to a site of embedded ideology.

The persistence of the figurative within the wider community ensures it relevance as an important architectural concern. The following quote by journalist, Miranda Devine, on figurative readings contained within the National Museum of Australia (NMA) by Melbourne Firm Ashton Raggatt McDougal (ARM) underscores this expectation for buildings to possess a narrative capacity. ...[T] he national identity portrayed by the museum was designed to make visitors hang their heads in shame.

As one museum council member said, it made "people leave the museum hating other Australians".

Little can be done about other attempts by the architects to falsely equate Aboriginal history with the Jewish Holocaust in Europe. The imagery is embedded in the design, copied in part from Daniel Libeskind's Jewish Museum in Berlin, which combines a broken Star of David with an SS symbol.

But, under Morton, what he calls the "black T-shirt" view of Australian culture is being replaced by something more complex and accurate.

"I want people to come out feeling good about Australia," he said... Devine [8].

Implicitly Devine accepts that representation, as an act of signification or naming, is one of architecture's 'jobs'. The question is not whether architecture can or should possess narrative facility but more an issue what it should be allowed to say. Architects may dismiss this as being unsophisticated but the context in which this expectation exists has a tendency to reassert itself irrespective of whether or not it is acknowledged in the conception, production 
or promotion of the artefact. Ultimately the failure to recognise this social expectation can only create a rupture between the profession and the community.

This should not be misconstrued as an argument for a return to figurative representation as a central architectural issue. The conventionalising of the tectonics of architecture to achieve semiotic fidelity between the sign and what it attempts to re-present traps the artefact's agency to the act of saying. This is particularly problematic given the mass media's capacity to configure new semiotic codes as a mechanism to propagate the specific political and commercial agendas of that minority controlling its production. After all selfinterest necessitates a thinking that replaces the complexities and contradictions of the social by universalising narratives. When Devine expresses the need for architecture, as a public act, to 'make good', the ensuing imagery serves only to normalise the ideological positions of those officially sanctioned narratives. The architect's complicity with this conceptually conservative framework extends beyond the issue of political censorship. Just as importantly the figurative, as a codified set of pre-inscribed signifiers, automatically limits all formal and spatial possibilities to a constant redrawing or re-presentation of these same signifiers. As such the figurative's 'job' of 'saying' or 'naming' evacuates representation, as an issue of both meaning and tool, of any generative experimental potential.

This is not to exclude the possibility that representation, particularly as an issue of signification, possessing a generative facility. In the essay Unreal Estate, prominent Australian architect, Carey Lyons, suggests that the strategies and techniques associated with the image's social and cultural function can operatively rethink the making of the built environment. To quote:

"The physical necessities of the industrial city...are being replaced by urban 'forces' that lack a physical nature: mass media, information technologies, a culture of consumption and services... This city of images, modern myths, and commodity spectacles is a strange and complex matrix of ephemera that are the conceptual and cultural substance with which to make our cities." Lyons [9].

It would be easy to dismiss this position as a simple extension of Venturi's promotion of the image's semiotic fidelity to that which it attempts to 'stand in' for. However, the essay's advocacy for the appropriation of imagistic strategies, tactics and techniques from the 'radical artifice' Lyons [10] of the contemporary social context offers the image productive value. Such strategies and techniques can be considered operative because its agency is focused on the production of alternatives more than the relating of some prescribed narrative. This thinking is evident when Lyon's writes that these “...strategies will need to take account of the qualities of these new ephemera with speculative methods and processes, which might create new types, new models and new readings of our cities." Lyons [11]. The use of the image is therefore inherently conceived for an ability to instigate a new range of urban and architectural solutions resulting from the forces shaping the contemporary context more than a preoccupation to convey specific codified 'messages'. Lyon's response to both image and context is therefore dramatically different to Lynn's indexing precisely because it understands its affect as a qualitative contextual rather than physically quantitative phenomenon. 
Yve Alain offers an insight into the way in which this performative framing of representation, as an issue of signification, could be approached. Of particular relevance is Bois's discussion, in Formless: A Users Guide, of Bataille's reading of Manet's Olympia, where both the painting's form and content create an "... uprooting, which he [Bataille] also calls a slippage, that... [reveals] Manet's "secret": the true goal of his art is to "disappoint expectation" Bois and Krauss [12]. The vital shift in the understanding of performance of representation occurs in the identification that the social conventionalising of the image's form and content also makes it implicitly susceptible to deliberate subversive slippages. The rejection of the image's role of 'naming' becomes operative because it is effectively an undoing of the conventional. As "...neither a theme, nor a substance, nor a concept..." Bois and Krauss [13]. Bataille's 'Informe' is antithetical to the attempt to create or stabilise meaning so that the instrumentality of formless resides in the capacity to operatively unsettle any ideological formations sublimated within the desire for the artefact to express figurative 'content' [14]. (Bois defines 'formless in the following way: "It is not so much a stable motif to which we can refer, a symbolizable theme, a given quality, as it is a term allowing one to operate a declassification, in the double sense of lowering and of taxonomic disorder. Nothing in and of itself, the 'formless' has only operational existence: it is a performative, like obscene words, the violence of which derives less from the semantics than from the very act of their delivery... The formless is an operation.")

In this light ARM's National Museum of Australia provides one way in which this operative undoing of figurative content might work. The project's strategic inundation and superimposition of multiple images and figures operatively resists the explication of a singular narrative. Devine's antagonism to the 'messages' within the N.M.A. can be understood as the subversion of the expectation for the building to deliver to the nation a politically sanctioned idealised image. Resonate with Carey Lyons' suggestion that public space should no longer be "...defined by the tradition of civitas, but as the medium through which the culture of mass media passes" Lyons [15], the project's tactical use of a disjunctive matrix of partial and complete two and three dimensional 'images' prises open representation to present the figurative without any unequivocal semiotic coding. As such the building acts against any paternalistic political structure by resisting its function to demonstrate the government's 'good works'. Also of import is that the generation of the design, through a mixture of image manipulation and modelling software, mediates the instrumentality of these digital tools through a socially responsive 'filter'. Issues of signification and of tool act together to establish contextual relevance.

Critically the project is more compelling where its 'images' and 'figures' are more ambiguous and unstable. Those design actions, which assume a strong semiotic correlation between image and meaning, risk marginalising the project by accusations of being too polemical. The issue extends beyond the question of whether this strategy is contextually relevant. If nothing else Devine's article demonstrates that the N.M.A.'s resistance to an easy cultural assimilation proves this point. The problem is an issue of tolerance. The capacity for these 
'messages' to be rewritten should not be underestimated. This much is evident in the changes to the original central courtyard and exhibition where the more sophisticated and complex reading of history have been replaced with an idealised and less challenging narrative. This strategy reveals its weakness because of its contingency on a suitable social and political context to commission and accept the project upon completion.

The capacity for the artefact to work within a less tolerant context instead relies on another performative potential, where the separation of any correlation between image and meaning allows an alternative approach to representation. In this respect the following quote from Alejandro Zaera Polo and Farshid Moussavi's discussion the Yokohama Ferry Terminal is instructive.

"When we won the Yokohama competition, we participated in several press conferences. They asked us "what was the idea of the project?" They looked at us strangely when we began to speak of diagrams, circulation and flows. Nobody seemed to understand anything. By the time of the fifth interview we remembered Hokusai's drawings and when they asked us for the generating idea, we let it out. There was an enormous "Aaahh!" of approval in the hall. Everyone understood immediately. Depending on the moment, you have to emphasize one thing or another" Zaera-Polo and Moussavi [16].

The analogy to the iconic figure of Hokusai's wave allows the public to engage with the project without any necessity for any associated figurative narrative or symbolism. As a formal reference, it is focused on the projection of an experiential quality rather than to narrative. The folding of the figure's generative facility also adds depth and direction to the diagrammatic analysis of temporal shifts of program without the resorting to or privileging of the instrumentality of animation software.

The instructive aspect of this discussion of the Yokohama Ferry Terminal is the way in which the performative potential of the projective image revolves around the difference between the figurative and figural. Deleuze, in his account of Francis Bacon's paintings, best articulates this distinction as the figurative being representational and "... implies the relationship of an object that it is supposed to illustrate: but it also implies a relationship of an image to other images in a composite whole that assigns a specific object to each of them" Deleuze [17]. On other hand the figural is released from this necessity to represent or to possess a narrative trajectory. Isolated from narrative "...the figure becomes an Image, an Icon... (it must)... stick to the fact." Deleuze [18]. Released from the 'job' of signification, the figure becomes performative because it frustrates meaning, allowing the object's material presence to exercise affect. This is case it is very different to the normative understanding of 'affect' as some type of extenuated temporal delay allowing the presence of the 'thing' to enforce itself to one's consciousness even after the object assumes intelligible meaning. Deleuze effectively challenges this temporal conception by arguing that it is Bacon's visual strategies that short-circuit to permanently 'put off' the possibility of the figurative forming meaning. This is important because the figural is an operative undoing that resists a conception of affect as a codified set of forms deployed to solicit specific experiential outcomes. 
At this point I would like to discuss the proceeding issues as they apply to my masters design research which explores the operative aspect of the image and in respect to representation and context. The project for RMIT University's (Royal Melbourne Institute of Technology University) new Janefield 'technology estate' on Melbourne's northern periphery departs from an appropriation the image of a stain that is strangely anthropomorphic. The campus form is made by, first, translating the figure into a vector line file, via Photoshop and lllustrator, and then importing it into Amapi, a three-dimensional modelling program. Once here the profile is deployed in two ways. It acts as a template for the campus 'plan' and for two different elevations, each accounting for the primary directions from which it can be viewed from ground level. The elevations are developed as anamorphic projections of oblique views taken from either side of the original image, fig. 1. These projections are then alternately spaced at regular intervals and rescaled proportionally within the 'plan' outline. A 'Gordon Surface' is extruded to generate the maximum volume of the scheme, which is then translated into architectonic form by a series of Boolean subtractions. These cuts are determined by programmatic and pragmatic requirements.
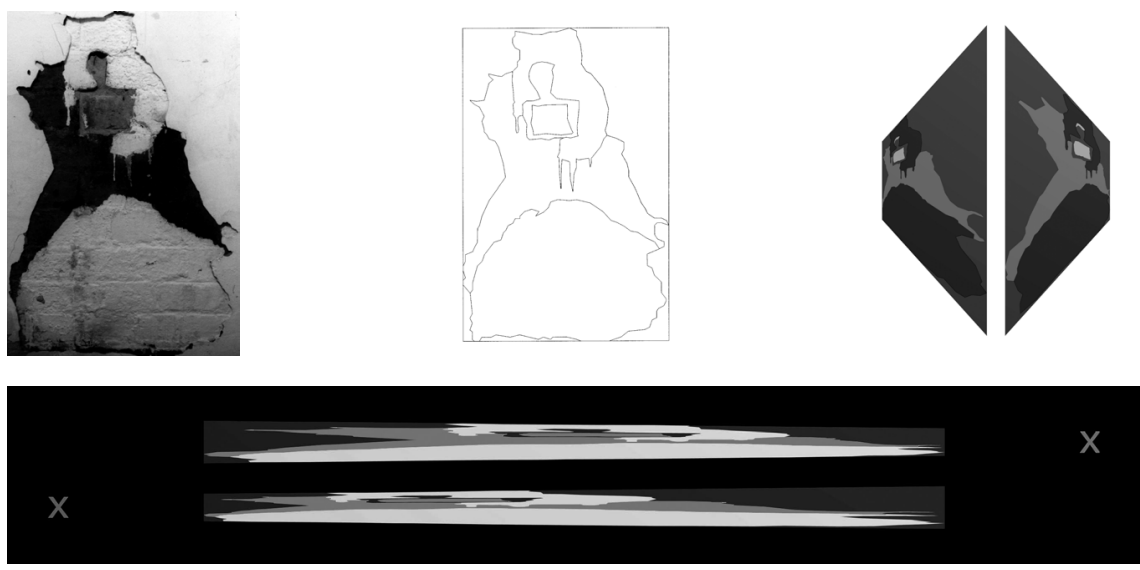

Figure 1: Translation of image to Anamorphic Projection.

The selection criterion for the figure was dictated by a lack of figurative or illustrative specificity. The figure becomes an enigmatic emblem that 'stands' for the body of the institution but can neither impart meaning nor tell its story. Without this representational stability the image works to both mark the absence of this possibility and reveal the conceit of the desire to do so. Standing in for the university, the figure acts only to locate the impossibility of satisfying this desire to re-present that which cannot actually exist. As Mark C. Taylor identifies this performance is an attempt to figure the 'unfigurable' Taylor [19]. (Mark C. Taylor argues that twentieth design culture has been preoccupied by the desire to disfigure and that modernism's abstraction and early post-modernism's 
excessive re-figuration participate in the same desire to capture and isolate the transcendental to make it real. Both see the relationship between signifier and the signified as absolute.)

The scheme can be seen to oscillate between the autonomy of the image and the pragmatic concerns of the project. These competing issues offer a mutual resistance preventing one concern from dominating the other. Superficially this is helpful in that both are satisfied by the requirement for pragmatically loose and mutable envelopes. The real import of this interaction, however, is that the interaction amplifies the affect of the figural reappearance at each cut, an occurrence exacerbated by the use of the figure to address a number of pragmatic concerns across a range of scales throughout the scheme. Operatively this creates an unpredictable eruption of the figure more akin to Bois and Krauss's notion of pulse, where the temporal discontinuity affects a sensation at each return. This is stark contrast to Lynn where the temporal exists only as an indexed set of numbers that are then processed to make form.

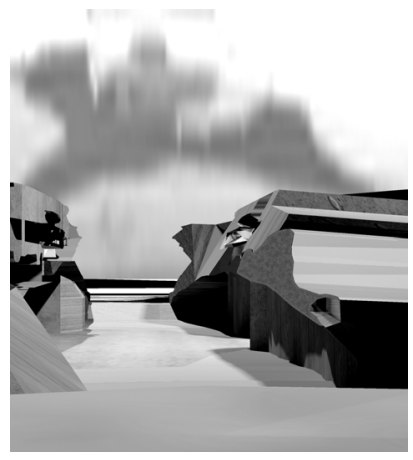

Figure 2: $\quad$ Experiential view.

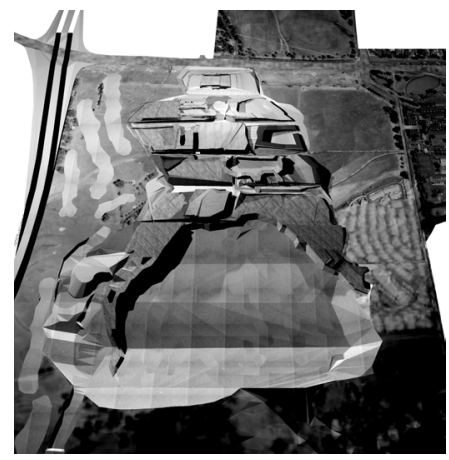

Figure 3: Aerial view of campus.

The scheme engages with the institutional desire to represent itself. The commercial imperative to generate funding streams coupled with the desire to attract students places an onus on the 'Institution' to distinguish itself. The image, as a form of currency, imparts such prestige to its buildings, which through their iconographic strength 'advertise' the institution. To RMIT, which has strategically employed the 'trophy building' as a form of promotion, this manoeuvre becomes a strategic imperative. The image of the figure aims to impart this distinction in a context where restricted budgets compromise the ability of the university to deliver the 'trophy building'. In doing so it takes the place of more traditional demonstrations of distinctiveness achieved through the use of geometry, materiality and detail. As such the appearance of the figure within the experiential or phenomenal view, as seen in figure 2 , is understood as only one of many possible views to acknowledge the strategic value of the iconic or emblematic view of the campus. In the first instance this would manifest itself in the form of the architect's speculative 'vision' of the completed master plan. As this image is subsumed by the built reality other vehicles are appropriated to ensure a public presence. This recognises both traditional modes 
and more contemporary virtual 'venues' ranging from the architectural publication and the postage stamp to new virtual 'sites' like Google Earth, fig. 3. For this reason the image 'in' the plan is as vital as those within the site.anefield scheme is conceivable only because of the existence of digital tools. These tools are significant because they allow design practice to revisit a range of architectural concerns. They also not only aid our understanding of context but they also shape it. The nature of the current political, economic and social contexts demonstrates the hazard of any withdrawal from an engagement with representation as an issue of signification or as a tool. To do so ensures that the artefacts remain ideologically trapped within a construction that it neither contests arguments of signification nor acknowledges the affect the tools of representation architecture's artefacts. The associated privileging of a narrow range of software acts only to prematurely close the potential of the digital as well as deny the reality that all 'drawing' renders some part of all design processes illustrative. Conversely, the critical acknowledgement of the generative potential of representation, and by association the image, as a response to context, opens the potential of representation to re-think the making of architecture's artefacts.

\section{References}

[1] Lynn, G., Animate Form, New York: Princeton Architectural Press, 1999, p.39.

[2] Mitchell, W.J.T. Iconology: Image, Text, Ideology, Chicago: University of Chicago Press, 1987, p.43

[3] Lynn, G., Animate Form, New York: Princeton Architectural Press, 1999, p.39.

[4] Lynn, G., Animate Form, New York: Princeton Architectural Press, 1999, p.39.

[5] This is also evident in the range of generative techniques Lynn uses throughout the book Animate Form.

[6] Evan's, R., Translations from Drawing to Building and Other Essays, Cambridge, Mass. : MIT Press, 1997, p 153-193.

[7] Rajchman, J., Constructions, Cambridge, Mass. : MIT Press, 1998, p. 5576.

[8] Devine, M., Disclosed At Last, The Embedded Messages That Adorn Museum, Sydney Morning Herald Online www.smh.com.au, April 2, 2006.

[9] Lyons, C., Unreal Estate, Take 1: Urban Solutions: Propositions for the future Australian City, ed. Robert McGauran, A.C.T., Australia: Royal Australian Institute of Architects, 2002.

[10] Lyons, C., Unreal Estate, Take 1: Urban Solutions: Propositions for the future Australian City, ed. Robert McGauran, A.C.T., Australia: Royal Australian Institute of Architects, 2002. 
[11] Lyons, C., Unreal Estate, Take 1: Urban Solutions: Propositions for the future Australian City, ed. Robert McGauran, A.C.T., Australia: Royal Australian Institute of Architects, 2002.

[12] Bois, Y. and Krauss, R., Formless: A Users Guide, Cambridge Mass.\& New York: M.I.T. Press \& Zone Books, 1997, page 15.

[13] Bois, Y. and Krauss, R., Formless: A Users Guide, Cambridge Mass.\& New York: M.I.T. Press \& Zone Books, 1997, page 15.

[14] Bois, Y. and Krauss, R., Formless: A Users Guide, Cambridge Mass.\& New York: M.I.T. Press \& Zone Books, 1997, page 18.

[15] Lyons, C., Unreal Estate, Take 1: Urban Solutions: Propositions for the future Australian City, ed. Robert McGauran, A.C.T., Australia: Royal Australian Institute of Architects, 2002.

[16] Zaera-Polo, A. and Moussavi, F., 2G: Revista Internacional De Arquitectura (International Architecture Review) number 16, Barcelona, Spain: G. Gili, 2000, p. 126

[17] Deleuze, G., The Logic of Sensation, transl. Smith, D.W., Minneapolis: University of Minnesota Press, 2004, p.6

[18] Deleuze, G., The Logic of Sensation, transl. Smith, D.W., Minneapolis: University of Minnesota Press, 2004, p.6

[19] Taylor, M.C., Disfiguring: Art, Architecture, Religion, Chicago: University of Chicago Press, 1992. 